\title{
Possible association between phantom vibration syndrome and occupational burnout
}

This article was published in the following Dove Press journal:

Neuropsychiatric Disease and Treatment

4 December 2014

Number of times this article has been viewed

\section{Chao-Pen Chen' \\ Chi-Cheng Wu ${ }^{2}$ \\ Li-Ren Chang ${ }^{3}$ \\ Yu-Hsuan Lin $^{4}$}

'Department of Education, National Taiwan University Hospital,

${ }^{2}$ Department of Family Medicine,

Min-Sheng General Hospital, Taoyuan

City, ${ }^{3}$ Department of Psychiatry,

National Taiwan University,

College of Medicine, ${ }^{4}$ Institute

of Brain Science, National Yang-Ming

University, Taipei, Taiwan
Correspondence: Yu-Hsuan Lin Institute of Brain Science, National Yang-Ming University, 177 Chung Cheng East Road, Section 2, Tamsui District, New Taipei City, Taiwan 25I

Tel +88655789317

Fax +886228097676

Email yuhsuanmed@gmail.com
Background: Phantom vibration syndrome (PVS) and phantom ringing syndrome (PRS) occur in many cell phone users. Previous studies have indicated an association between PVS/PRS and job stress. The aim of this study was to determine if PVS/PRS were also associated with occupational burnout.

Methods: This was a cross-sectional study of 384 employees of a high-tech company in northern Taiwan. They all completed a phantom vibration and ringing questionnaire, the Hospital Anxiety and Depression Scale, and the Chinese version of the Occupational Burnout Inventory.

Results: Significantly more women and people with at least a college education were in the population with PRS and PVS, respectively. Anxiety and depression had no associations with PVS/PRS. Higher scores for personal fatigue, job fatigue, and service target fatigue had an independent impact on the presence of PVS, but only a higher score for service target fatigue had an independent impact on the presence of PRS.

Conclusion: The independent association between work-related burnout and PVS/PRS suggests that PVS/PRS may be a harbinger of mental stress or a component of the clinical burnout syndrome, and may even be a more convenient and accurate predictor of occupational burnout.

Keywords: phantom vibration syndrome, phantom ringing syndrome, occupational burnout

\section{Introduction}

Worldwide, an increasing number of people carry cell phones and utilize the vibration mode to ensure silence in quiet areas. Extensive use of this mode may be associated with the perception that the phone is vibrating when it is not, ie, the phantom vibration syndrome (PVS). A 2010 study by Rothberg et $\mathrm{al}^{1}$ found that $68 \%$ of the medical staff at an acute care hospital had experienced PVS. Factors associated with PVS were occupation (residents versus attending physicians), location of phone (breast pocket versus belt), number of hours carried, and amount of time in vibrating mode. The phantom ringing syndrome (PRS) has also been described, but occurs less frequently. PVS and PRS may be "hallucinations" but they are not psychoses. A meta-analysis by Waters et al proposed a general model that includes signal detection errors, executive and inhibition deficits, a tapestry of expectations and memories, and state characteristics that influence how these experiences are interpreted. ${ }^{2}$ Nonpsychotic "hallucinations" have been described in bereavement (deceased spouse) ${ }^{3}$ and in post-traumatic stress disorder (re-experienced trauma). ${ }^{4}$ A previous study by Lin et al focused on PVS/PRS in medical interns, a defined population of a similar age, lifestyle, and educational background exposed to a timelimited stressor. ${ }^{5}$ In an aroused and hypervigilant state, the interns anticipated an emergency summons and misinterpreted sensory input from another source. ${ }^{6}$ Lin et $\mathrm{al}^{7}$ found that $78 \%$ of medical interns experienced PVS while only $27 \%$ experienced PRS before the stressful internship. Both PVS and PRS increased dramatically over the course of 
the stressful internship (86.7 hours/week, 33.5 consecutive work hours, and 10 on-call duties per month) and decreased after it was completed. Interns with severe PVS or PRS showed a significant increase in anxiety and depression ${ }^{5}$ as well as reduced sympathetic modulation and persistent inattention. ${ }^{8}$ Neurodevelopmental changes associated with the hypothalamic-pituitary-adrenal axis have previously been investigated as mechanisms for auditory hallucinations among children who have experienced trauma ${ }^{9}$ and post-traumatic stress disorder. ${ }^{10,11}$ Our previous study also showed that the heavy workload during an internship resulted in long-term and short-term alternations in autonomic nervous system modulation. ${ }^{4,8,12}$ The increase in PVS and/or PRS appeared to parallel the level of stress experienced by the interns.

Selye et al ${ }^{13}$ had proposed the "general adaptation syndrome" model to explain how the body responds to stress, which include three phases of stress response, ie, alarm stage, resistance stage, and exhaustion stage. "Occupational burnout" is another response to stress and is generally described as long-term exhaustion and disinterest in work. Kristensen et $\mathrm{al}^{14}$ considered three domains, ie, personal burnout, work-related burnout, and client-related burnout. Although anxiety, depression, and somatic symptoms are often associated with burnout, opinions differ as to whether these represent long-term psychopathology ${ }^{15}$ or environmental factors such as work load ${ }^{16}$ or lack of psychosocial safety. ${ }^{17}$ Employment insecurity, a perceived lack of workplace justice, loss of control, and lack of support have also been suggested as contributors. ${ }^{18,19}$

Most previous studies have addressed the epidemiology, clinical characteristics, or social impact of burnout. The association of burnout with PVS/PRS has not been investigated previously, especially in nonmedical professionals. The exhaustion and fatigue associated with burnout may similarly predispose individuals to misinterpretation of sensory input. The present study examined the prevalence of PVS/PRS in a cross section of employees in a high-tech company with chronic stress and various degrees of occupational burnout, manifesting primarily as fatigue.

Our aim was to evaluate the association of occupational burnout with PVS and PRS in mobile phone users in the regular workplace. We hypothesized that PVS and PRS would be related to burnout as would depression and anxiety.

\section{Materials and methods Participants}

We recruited 391 employees of a high-tech company in northern Taiwan. They were asked to complete self-reported questionnaires in March 2011 and 384 (98\%) returned them. All the questionnaires were self-reported voluntarily; however, not all subjects answered every question. Subjects had been informed that participation in the survey was completely voluntary, confidentiality was assured, and that the research ethics committee of the National Taiwan University Hospital had approved this study prior to implementation.

\section{Measurements}

\section{Phantom vibration and ringing questionnaire}

To avoid biasing the respondents, the questionnaire simply stated: "We are asking you to participate in a research study survey about cell phones because, in your job, you carry one." The questions included whether the respondent had experienced phantom vibration or phantom ringing during the previous 3-month period, as well as potential factors associated with phantom vibration that had been documented in a previous cross-sectional study, ${ }^{1}$ ie, whether the device was used in vibration or ringing mode and where it was worn. Those who reported phantom vibration or phantom ringing were also asked how bothersome these events were. These were both scored on 5-point Likert scales $(0-4)$ where any score over 0 was positive.

\section{Hospital Anxiety and Depression Scale}

The Hospital Anxiety and Depression Scale (HADS) includes seven items that form the anxiety subscale (HADS-anxiety) and seven that form the depression scale (HADS-depression); it does not include any somatic symptoms. ${ }^{20}$ Each item was scored on a 4-point Likert scale, with higher scores indicating a greater degree of anxiety or depression. Example items were "I feel nervous and my nerves are on edge" and "I feel a lack of interest in my appearance". HADS has been widely used in general populations other than hospital inpatients.

\section{Occupational Burnout Inventory}

The Chinese Occupational Burnout Inventory (OBI) is a selfadministered questionnaire with 21 items and was modified from the Copenhagen Burnout Inventory. ${ }^{12}$ The OBI includes four subscales, ie, personal burnout, work-related burnout, client-related burnout, and overcommitment to work. Each item was scored on a 5-point Likert scale, with higher scores indicating a greater degree of burnout. An example item was "Are you exhausted in the morning at the thought of another day of work?" The reliability and validity were fair. ${ }^{21}$

\section{Statistical analysis}

Subjects were divided into PVS, PRS, and "PVS or PRS" groups for analysis. Previous research ${ }^{3}$ had shown that longer 
work periods have a stronger association with both PVS and PRS. These subjects have worked for several years, so a combined group was included in the analysis. Continuous data were presented as the mean \pm standard deviation and comparisons between groups were performed using independent two sample $t$-tests. Ordinal data were presented as the median and interquartile range and comparisons between groups were made using the nonparametric Mann-Whitney test. Categorical data were presented as numbers (percentages) and the associations among the categorical variables and the PVS and PRS groups were calculated using Fisher's Exact test. Correlations between HADS and OBI were assessed with Spearman's correlation coefficients $(\rho)$. Univariate and multivariate logistic regression analyses were utilized to investigate the associations of subject characteristics, HADS, and OBI with PRS, PVS, and "PRS or PVS". All statistical assessments were two-tailed and $P<0.05$ was considered to be statistically significant. Statistical analyses were performed using Statistical Package for the Social Sciences version 15.0 software (SPSS Inc, Chicago, IL, USA).

\section{Results}

\section{Subject characteristics}

The 384 subjects had an average age of $33.7 \pm 7.7$ years, 209 (54.4\%) were males, and most (65.6\%) had an educational level of college or above. Their median duration of work in the company was 5 years (interquartile range $2.5-10.7$ years), with an average of 9.3 working hours per day and 46.5 hours per week.

Most of the subject characteristics were not significantly associated with PVS or PRS, except for sex and level of education. Significantly more females were in the population with PRS than those without it (59.6\% versus $42.4 \%$, $P=0.024$ ), and significantly more subjects in the population with PVS had a level of education of college or above than did those without it $(77.5 \%$ versus $62.7 \%, P=0.016)$. In addition, significantly more subjects in the population with either PVS or PRS had a level of education of college or above than did those without PVS or PRS $(76.3 \%$ versus $62.9 \%, P=0.017$, Table 1).

\section{HADS and OBI versus PVS and PRS}

The HADS scores for anxiety and depression between PVS and non-PVS subjects, PRS subjects and non-PRS subjects, or subjects with either PVS or PRS and those without either (median scores for anxiety and depression between PVS and non-PVS subjects, 8.0 versus 8.0 and 7.0 versus 8.0 ; between PRS and non-PRS subjects, 7.0 versus 8.0 and 7.0 versus 7.0;

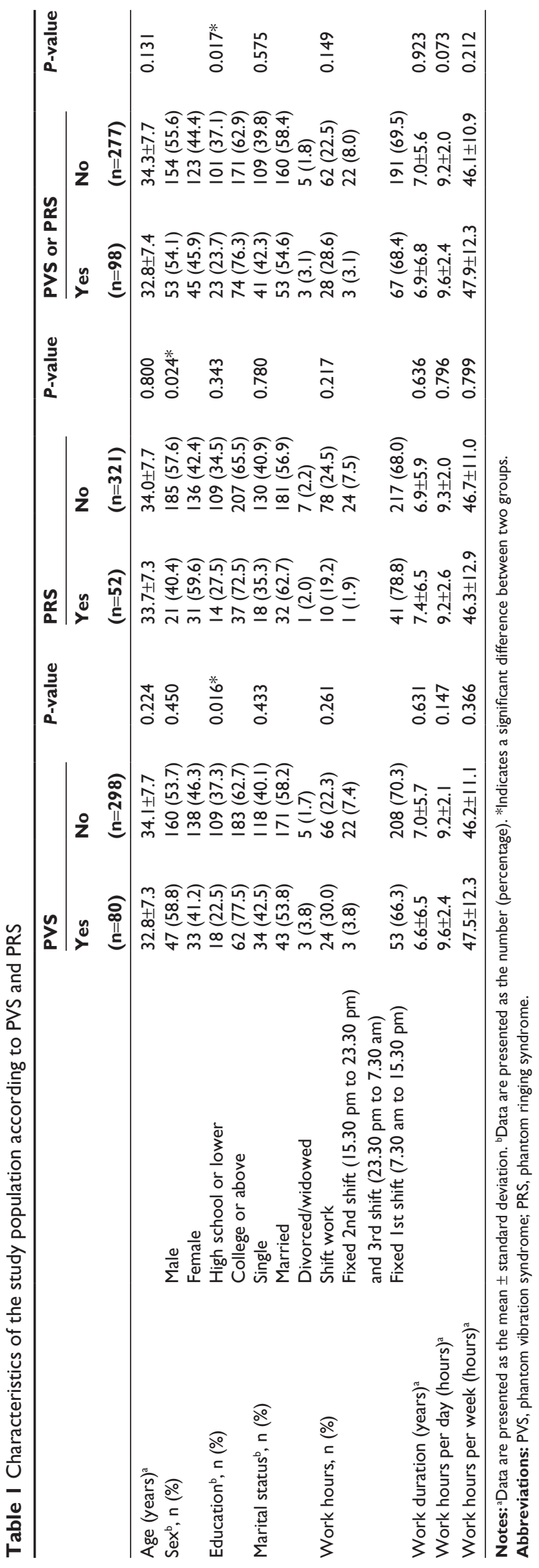


between either PVS or PRS subjects and those without, 8.0 versus 8.0 and 7.0 versus 8.0 ) did not differ significantly (all $P>0.05$, Table 2).

In terms of occupational burnout categories, the subjects with PVS had significantly higher scores for personal fatigue (median 50.0 versus $40.0, P=0.001$ ), job fatigue (median 50.0 versus 40.0, $P=0.008$ ), and job overcommitment (median 45.0 versus 35.0, $P=0.035$ ). Subjects with either PVS or PRS had significantly higher scores for personal fatigue (median 50.0 versus $40.0, P=0.004$ ) and job fatigue (median 50.0 versus 40.0, $P=0.016$; Table 2).

\section{OBI versus HADS}

The Spearman correlation coefficients $(\rho)$ presented in Table 3 showed weak to moderately positive correlations between OBI and HADS ( $\rho$ ranged from 0.272 to 0.549 ). Personal fatigue and job fatigue were moderately correlated with anxiety and depression ( $\rho$ 0.4-0.6), job overcommitment was weakly correlated with anxiety and depression ( $\rho$ 0.2-0.4), and service target fatigue was moderately correlated with anxiety and weakly correlated with depression.

\section{Independent associations of $\mathrm{OBI}$ with PVS and PRS}

Level of education was found to be associated with PVS (odds ratio [OR] 2.05, $P=0.014$ ) and "PVS or PRS" (OR 1.90, $P=0.017$ ), and sex was found to be associated with PRS (OR 2.01, $P=0.022$ ). HADS scores for both anxiety and depression were not significantly associated with PVS or PRS; however, some domains of OBI were significantly associated with PVS and "PVS or PRS", so further multivariable analyses were performed to evaluate the independence of the associations of OBI with PVS and PRS (Table 4).

All of the ORs for PVS or PRS were adjusted for age, sex, and education. Higher scores for personal fatigue (OR 1.03, $P=0.001$ ), job fatigue (OR 1.03, $P=0.001$ ), and service target fatigue (OR 1.03, $P<0.001$ ) had an independent impact on the presence of PVS, but only a higher score for service target fatigue had an independent impact on the presence of PRS (OR 1.02, $P=0.016$ ). Higher scores for personal fatigue (OR 1.02, $P=0.006$ ), job fatigue (OR 1.02, $P=0.003$ ), and service target fatigue (OR 1.02, $P=0.001$ ) had an independent impact on the presence of either PVS or PRS (Table 5).

\section{Discussion}

PVS and PRS occur for many cell phone users. We found an independent association between PVS and PRS and occupational burnout in this group of high-tech employees.

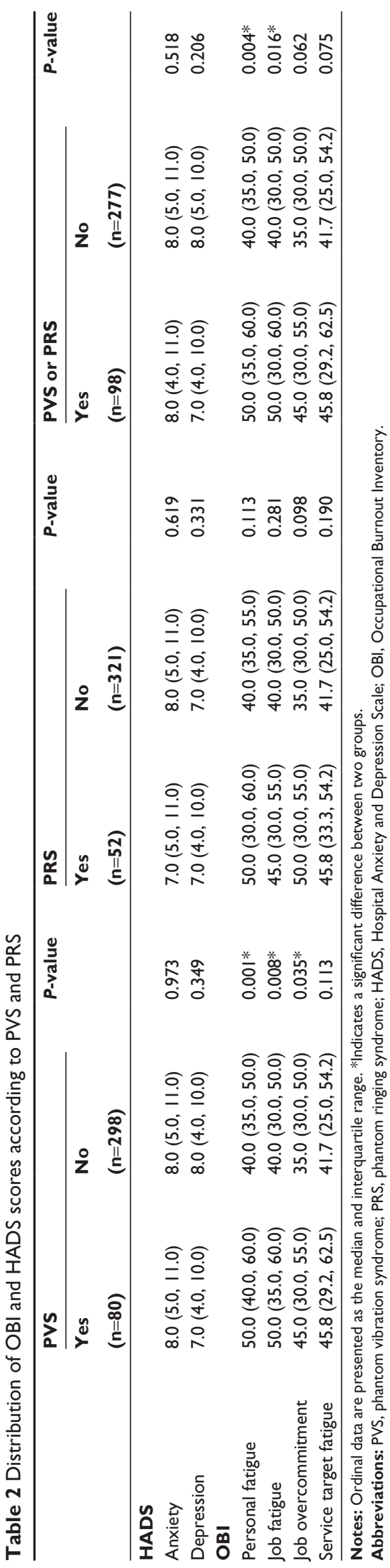


Table 3 Correlations between OBI and HADS

\begin{tabular}{|c|c|c|c|c|}
\hline & & & \multicolumn{2}{|c|}{ HADS $(n=384)$} \\
\hline & & & Anxiety & Depression \\
\hline \multirow[t]{8}{*}{ OBI $(n=376)$} & Personal fatigue & Spearman's $\rho$ & 0.549 & 0.443 \\
\hline & & $P$-value & $<0.001$ & $<0.001$ \\
\hline & Job fatigue & Spearman's $\rho$ & 0.477 & 0.406 \\
\hline & & $P$-value & $<0.001$ & $<0.001$ \\
\hline & Job overcommitment & Spearman's $\rho$ & 0.324 & 0.272 \\
\hline & & $P$-value & $<0.001$ & $<0.001$ \\
\hline & Service target fatigue & Spearman's $\rho$ & 0.410 & 0.318 \\
\hline & & $P$-value & $<0.001$ & $<0.001$ \\
\hline
\end{tabular}

Abbreviations: HADS, Hospital Anxiety and Depression Scale; OBI, Occupational Burnout Inventory.

This provides a model for a stress-induced psychosis rather than childhood trauma or biological illness. ${ }^{22}$

In the present study, there were significantly more women in the population with PRS and those with at least a college education were represented more often in the population with PVS. Dewi Rees reported no difference by sex among the widowed, and that the "professional and managerial" group of widows and widowers was more likely to "hallucinate" than "nonmanual and sales workers". ${ }^{3}$ This sex and occupation disparity requires further study.

Anxiety and depression were not associated with PVS/ PRS in the present study. However, without a dimensional approach to PVS/PRS in this study and few severe PVS/ PRS cases, we cannot rule out specific correlations between anxiety, depression, and PVS/PRS. In the study by Lin et $\mathrm{al}^{5}$ interns experiencing severe phantom ringing were more depressed than interns experiencing subclinical phantom ringing. The correlation of higher cognitive/affective depressive scores in interns with severe phantom vibration and phantom ringing enhances the viewpoint that phantom vibration is synthesized through a cognitive mechanism. More specifically, our results suggest that phantom ringing, an auditory hallucination, is more relevant to a catastrophic cognitive formulation than is phantom vibration.

Among the current subjects, higher scores for personal fatigue, job fatigue, and service target fatigue had an independent impact on the presence of PVS, but only a higher score for service target fatigue had an impact on the presence of PRS. Burnout due to fatigue may be less catastrophic and may also respond to nonpharmacological treatment such as exercise training. ${ }^{23}$

Based on the hypothesis that phantom vibration and phantom ringing are transient novel responses, the present study is consistent with the well-known overlapping circuits in the limbic forebrain, hypothalamus, and brainstem that mediate stress responses, emotional learning, and reward processing. ${ }^{24}$ Menke et $\mathrm{al}^{25}$ found glucocorticoid receptorinduced neuroendocrine and gene expression changes in men suffering from job-related exhaustion. These changes returned to normal after the men recovered. Verhaeghe et $\mathrm{al}^{26}$ noted that burnout was associated with hypofunction of the hypothalamic-pituitary-adrenal axis, a neurocharacteristic of exhaustion, and this supports a possible relationship with PVS/PRS.

There are limitations to this study. No reliable data exist about the prevalence of PVS/PRS in the general population. Subjects were employed by a single company and personality characteristics and job responsibilities were not taken into account. Personal or work-related stressors were not identified.

There are two major methodological limitations that should be noted when interpreting our findings. First, the cross-sectional design using a convenient sample from a high-tech company in this study limits our ability to make causal inferences on the relationship between PVS/PRS and its correlates, and also limits generalization. Second, the data were derived from self-reported assessments rather than direct diagnostic interviews. A more comprehensive longitudinal study design is needed to validate the phenomena identified in this study and to explore the underlying mechanisms further.

\section{Conclusion}

As a pilot study in this field, our results provide new insights into the occupational burnout associated with PVS/PRS. The independent association of occupational burnout and PVS/ PRS suggests that PVS/PRS may be a harbinger of mental stress or a component of the clinical burnout syndrome. A simple question about experience with an electronic device may be less intrusive, and itself generate less anxiety than questionnaires identified as being about stress and burnout. This may also prove to be more convenient and to produce 


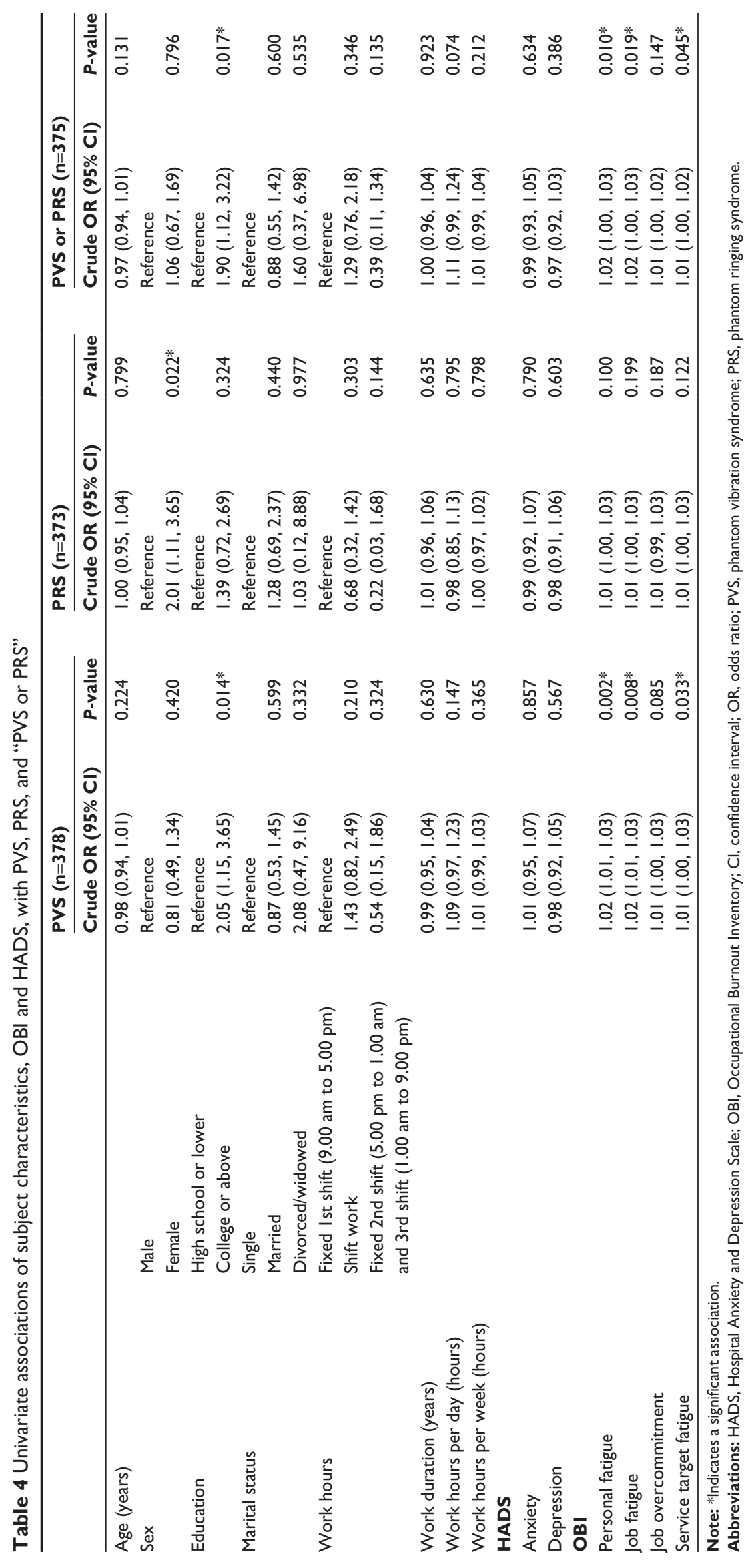


Table 5 Multivariate associations of OBI with PVS, PRS, and "PVS or PRS"

\begin{tabular}{|c|c|c|c|c|c|c|}
\hline & \multicolumn{2}{|l|}{ PVS $(n=378)$} & \multicolumn{2}{|l|}{ PRS (n=373) } & \multicolumn{2}{|l|}{ PVS or PRS $(n=375)$} \\
\hline & Adjusted OR $(95 \% \mathrm{Cl})$ & $P$-value & Adjusted OR $(95 \% \mathrm{Cl})$ & $P$-value & Adjusted OR $(95 \% \mathrm{Cl})$ & $P$-value \\
\hline \multicolumn{7}{|l|}{$\overline{O B I}$} \\
\hline Personal fatigue & $1.03(1.01,1.04)$ & $0.001 *$ & $1.01(1.00,1.03)$ & 0.133 & $1.02(1.01,1.03)$ & $0.006 *$ \\
\hline Job fatigue & $1.03(1.01,1.04)$ & $<0.00 I^{*}$ & $1.02(1.00,1.03)$ & 0.098 & $1.02(1.01,1.04)$ & $0.003 *$ \\
\hline Job overcommitment & $1.01(0.99,1.03)$ & 0.251 & $1.01(0.99,1.03)$ & 0.392 & $1.01(0.99,1.02)$ & 0.353 \\
\hline Service target fatigue & $1.03(1.01,1.04)$ & $<0.001 *$ & $1.02(1.00,1.04)$ & $0.016 *$ & $1.02(1.01,1.04)$ & $0.00 I^{*}$ \\
\hline
\end{tabular}

Notes: \#Adjusted for age, sex, and education. *Indicates a significant association.

Abbreviations: HADS, Hospital Anxiety and Depression Scale; OBI, Occupational Burnout Inventory; Cl, confidence interval; OR, odds ratio; PVS, phantom vibration syndrome; PRS, phantom ringing syndrome.

more accurate results, since there would be no need to "cover up" this experience. A further prospective, longitudinal cohort study may clarify this.

\section{Author contributions}

C-PC: literature research, data analysis, statistical analysis, and manuscript preparation. C-CW: study concepts, study design, definition of intellectual content, and data acquisition. L-RC: study concepts and data acquisition. Y-HL: guarantor of the integrity of the study, manuscript editing, and manuscript review. All authors contributed toward data analysis, drafting and revising the paper and agree to be accountable for all aspects of the work.

\section{Disclosure}

The authors report no conflicts of interest in this work.

\section{References}

1. Rothberg MB, Arora A, Hermann J, Kleppel R, St Marie P, Visintainer P. Phantom vibration syndrome among medical staff: a cross sectional survey. BMJ. 2010;341:c6914.

2. Waters F, Allen P, Aleman A, et al. Auditory hallucinations in schizophrenia and nonschizophrenia populations: a review and integrated model of cognitive mechanisms. Schizophr Bull. 2012;38:683-693.

3. Dewi Rees W. The hallucinations of widowhood. BMJ. 1971;4:37-41.

4. Mueser KT, Butler RW. Auditory hallucinations in combat-related posttraumatic stress disorder. Am J Psychiatry. 1987;144:299-302.

5. Lin YH, Chen CY, Li P, Lin SH. A dimensional approach to the phantom vibration and ringing syndrome during medical internship. J Psychiatr Res. 2013;47:1254-1258.

6. Baillie DWH. Sixty eight percent of us hallucinate. BMJ. 2011;342: d299.

7. Lin YH, Lin SH, Li P, Huang WL, Chen CY. Prevalent hallucinations during medical internships: phantom vibration and ringing syndromes. PLoS One. 2013;8:e65152.

8. Lin YH, Kuo TBJ, Ho YC, Lin SH, Liu CY, Yang CC. Physiological and psychological impacts on male medical interns during on-call duty. Stress. 2012;15:21-30

9. Cotter D, Pariante CM. Stress and the progression of the developmental hypothesis of schizophrenia. Br J Psychiatry. 2002;181:363-365.

10. Steel C, Haddock G, Tarrier N, Picken A, Barrowclough C. Auditory hallucinations and posttraumatic stress disorder within schizophrenia and substance abuse. J Nerv Ment Dis. 2011;199:709-711.
11. Wilcox J, Briones D, Suess I. Auditory hallucinations, posttraumatic stress disorder, and ethnicity. Compr Psychiatry. 1991;32:320-323.

12. Lin $\mathrm{YH}, \mathrm{Chen} \mathrm{CY}$, Lin $\mathrm{SH}$, et al. Gender differences in cardiac autonomic modulation during medical internship. Psychophysiology. 2013;50:521-527.

13. Selye H. Stress and the general adaptation syndrome. BMJ. 1950;1: 1383-1392.

14. Kristensen TS, Borritz M, Villadsen E, Christensen KB. The Copenhagen Burnout Inventory: a new tool for the assessment of burnout. Work Stress. 2005;19:192-207.

15. Rössler W, Hengartner MP, Ajdacic-Gross V, Angst J. Predictors of burnout: results from a prospective community study. Eur Arch Psychiatry Clin Neurosci. June 14, 2014. [Epub ahead of print].

16. Wang S, Liu Y, Wang L. Nurse burnout: personal and environmental factors as predictors. Int J Nurs Pract. November 15, 2013. [Epub ahead of print].

17. Idris MA, Dollard MF, Yulita. Psychosocial safety climate, emotional demands, burnout, and depression: a longitudinal multilevel study in the Malaysian private sector. J Occup Health Psychol. 2014;19:291-302

18. Cheng Y, Luh WM, Guo YL. Reliability and validity of the Chinese version of the Job Content Questionnaire in Taiwanese workers. Int J Behav Med. 2003;10:15-30.

19. Cheng Y, Huang HY, Li PR, Hsu JH. Employment insecurity, workplace injustice and employees' burnout in Taiwanese employees: a validation study. Int J Behav Med. 2011;18:391-401.

20. Zigmund AS, Snaith RP. The Hospital Anxiety and Depression Scale Acta Psychiatr Scand. 1983;67:361-370.

21. Yeh WY, Cheng Y, Chen CJ, Hu PY, Kristensen TS. Psychometric properties of the Chinese version of Copenhagen Burnout Inventory among employees in two companies in Taiwan. Int J Behav Med. 2007; 14:126-133.

22. Read J, Van Os J, Morrison AP, Ross CA. Childhood trauma, psychosis, and schizophrenia: a literature review with theoretical and clinical implications. Acta Psychiatr Scand. 2005;112:330-350.

23. Gerber M, Brand S, Elliot C, Holsboer-Trachsler E, Puhse U, Beck J. Aerobic exercise training and burnout: a pilot study with male participants suffering from burnout. BMC Res Notes. 2013;6:78.

24. Ulrich-Lai YM, Herman JP. Neural regulation of endocrine and autonomic stress responses. Nat Rev Neurosci. 2009;10:397-409.

25. Menke A, Arloth J, Gerber M, et al. Dexamethasone stimulated gene expression in peripheral blood indicates glucocorticoid-receptor hypersensitivity in job-related exhaustion. Psychoneuroendocrinology. 2014;44:35-46.

26. Verhaeghe J, Van Den Eede F, Van Den Ameele H, Sabbe BG. [Neuroendocrine correlates of burnout]. Tijdschr Psychiatr. 2012;54:517-526. Dutch. 


\section{Publish your work in this journal}

Neuropsychiatric Disease and Treatment is an international, peerreviewed journal of clinical therapeutics and pharmacology focusing on concise rapid reporting of clinical or pre-clinical studies on a range of neuropsychiatric and neurological disorders. This journal is indexed on PubMed Central, the 'PsycINFO' database and CAS,

and is the official journal of The International Neuropsychiatric Association (INA). The manuscript management system is completely online and includes a very quick and fair peer-review system, which is all easy to use. Visit http://www.dovepress.com/testimonials.php to read real quotes from published authors.

Submit your manuscript here: http://www.dovepress.com/neuropsychiatric-disease-and-treatment-journal 